\title{
Agentes hemostáticos en cirugía periapical. Revisión de literatura
}

\author{
Hemostatic agents in apical surgery. A review \\ Hernán Coaguila Llerena ${ }^{1, a}$, Carlos Mendiola Aquino ${ }^{2, b, c}$
}

\section{RESUMEN}

La zona periapical es una región altamente vascularizada, y exhibe una gran tendencia a la hemorragia, especialmente en presencia de tejido granulomatoso, que suele acompañar las patologías perirradiculares. Esto representa para el clínico un aspecto de suma importancia, ya que solo mediante un campo seco se obtendrá un campo operatorio efectivamente visible que le permita intervenir adecuadamente. Es necesario evaluar consideraciones pre-quirúrgicas y quirúrgicas tales como la historia médica del paciente, así como la manipulación de los tejidos antes de la elección del agente hemostático que se opte por conveniente. Los agentes hemostáticos presentan características que los hacen particularmente muy distintos unos de otros, tanto en su presentación como en su manejo operatorio. La presente revisión bibliográfica describirá y analizará los principales agentes hemostáticos que se encuentran actualmente disponibles en el mercado y su impacto en la cirugía periapical contemporánea.

PALABRAS CLAVE: endodoncia, procedimientos quirúrgicos orales, apicectomía, hemostasis, hemostáticos. (Fuente: DeCS, Bireme)

\section{SUMMARY}

The apical area is a highly vascular region, and exhibits a tendency to hemorrhage, especially in presence of granulation tissue that usually is present in periradicular pathologies. This is highly important for the clinician, because only with a dry field can be obtained a visible operative field that allows a suitable surgery. It is necessary to assess pre-surgical and surgical considerations such as medical history, as well as tissue manipulation before the election of a hemostatic agent. Hemostatic agents have characteristics that make them particularly very different from each other, both presentation and clinical management. The aim of this review is to describe and discuss the most common hemostatic agents available and their impact on contemporary apical surgery.

KEYWORDS: endodontics, oral surgical procedures, apicoectomy, hemostasis, hemostatics. (Source: MeSH, NLM)

Facultad de Estomatología, Universidad Peruana Cayetano Heredia. Lima, Perú.

Departamento Académico de Clínica Estomatológica, Universidad Peruana Cayetano Heredia. Lima, Perú.

Especialista en Endodoncia.

Docente

Past Presidente de la Sociedad Peruana de Endodoncia 


\section{INTRODUCCIÓN}

Cuando en los tratamientos endodónticos convencionales no se obtiene el éxito esperado la cirugía periapical suele estar indicada. En el acto quirúrgico, después de la resección del ápice, es necesario preparar una cavidad que recibirá un material de obturación retroapical (1). Independientemente del método y material utilizado para sellar la preparación retroapical, la obtención de una buena visión así como un campo operatorio seco es fundamental $(2,3)$. Una inapropiada hemostasia representa una seria dificultad durante la cirugía periapical, y se convierte en un punto crítico durante la preparación y más aún durante la obturación retroapical (4).

La zona periapical es una región altamente vascularizada que exhibe una gran tendencia a la hemorragia, especialmente en presencia de tejido granulomatoso que suele acompañar una patología periradicular (5). Por otra parte, el hueso alveolar está atravesado por vasos nutricios que están localizados deforma aleatoria, y muy a menudo pueden romperse durante el legrado, ocasionando hemorragia (1).

La historia clínica del paciente debe permitir una evaluación en detalle de sus condiciones pre-operatorias antes de ser intervenido quirúrgicamente, del mismo modo, un conocimiento práctico del proceso fisiológico de la coagulación sanguínea constituye un aspecto primordial para los clínicos que manejen la cirugía periapical (6).

\section{Consideraciones prequirúrgicas}

La historia clínica médica-odontológica debe considerar una minuciosa anamnesis, la cual permitirá una evaluación pormenorizada del paciente a través de los antecedentes fisiológicos y patológicos, alergias conocidas, y los fármacos que tome; incluyendo la naturaleza de la medicación, tanto la prescrita como la automedicada a fin de detectar la presencia o magnitud de alguna alteración de la hemostasia $(6,7)$.

Muchos medicamentos como la aspirina alterarán el mecanismo de coagulación a través de cambios en el equilibrio entre el tromboxano $\mathrm{A} 2\left(\mathrm{TXA}_{2}\right)$ sintetizado en las plaquetas y la prostaciclina $\left(\mathrm{PGI}_{2}\right)$ generada en la pared del vaso. La aspirina produce una inhibición irreversible de ciclooxigenasa por acetilación de un residuo de serina en las enzimas activas. Sin embargo, con este fármaco tanto el $\mathrm{TXA}_{2}$ y $\mathrm{PGI}_{2}$ se ven afectados ya que las células en las paredes de los vasos pueden producir ciclooxigenasa en cuestión de horas, mientras que las plaquetas son incapaces de producir esta enzima (7). El TXA 2 promueve la vasoconstricción y la agregación plaquetaria, mientras que la $\mathrm{PGI}_{2}$ inhibe la agregación plaquetaria y la vasodilatación (6).

En algunos casos, la premedicación con vitamina K1 (fitomenadiona) u otros hemostáticos sistémicos como el etamsilato, pueden ser útiles. (1)

Los signos vitales del paciente, tales como la presión arterial, la frecuencia cardíaca y la frecuencia respiratoria también deben ser evaluados, ya que la hipertensión y la taquicardia pueden indicar una patología cardíaca no diagnosticada u otras condiciones sistémicas (8). Los signos vitales también pueden ser utilizados para monitorear al paciente ansioso. Los aumentos en la presión arterial y la frecuencia cardíaca por encima de los valores normales conocidos también son indicadores de niveles de estrés elevados (6).

Es importante considerar que la ansiedad y estrés del paciente también pueden ocasionar sangrado y aumento de la fibrinólisis, y esto puede controlarse a través de la sedación, la cual estará supeditada a la historia médica-odontológica antes del procedimiento quirúrgico, de tal manera se disminuirá el elevado gasto cardiaco durante la intervención $(6,7)$.

\section{Consideraciones quirúrgicas}

El factor más importante de la hemostasia quirúrgica es el cirujano (9).

La atención al detalle en todo el procedimiento limitará los potenciales problemas de hemorragia. La cirugía periapical implica la manipulación de los tejidos blandos y cantidades variables de hueso cortical y esponjoso. Por lo tanto, la capacidad de controlar todas las etapas del proceso quirúrgico es esencial $(1,3)$. El uso de distintas técnicas anestésicas, el diseño y elevación del colgajo, y la eliminación de hueso puede afectar en gran medida la capacidad de obtener y mantener la hemostasia durante todo el procedimiento quirúrgico (6).

\section{Anestesia local}

La capacidad para lograr una anestesia profunda y operar dentro de la duración de su acción se relacionará directamente con el nivel de confort del paciente 
y la hemostasia en el campo quirúrgico. La mayoría de los anestésicos locales contiene un agente vasoconstrictor para mejorar el efecto de la anestesia (10). El aumento de la vasoconstricción local disminuirá el flujo de sangre a la zona quirúrgica, y también la disminución de la velocidad a la que el anestésico pierde el efecto. La combinación de lidocaína al $2 \%$ con adrenalina 1:100 000 complementado con lidocaína al 2\% con adrenalina 1:50 000 es considerado el protocolo actual (11). La lidocaína con adrenalina 1:50 000 actúa sobre los vasos sanguíneos del periostio, submucosa, encía y periodonto para producir vasoconstricción (6).

El uso de adrenalina 1:50000 permite una clara visualización de la cripta ósea, una reducción del tiempo quirúrgico, y una disminución de la hemorragia postoperatoria (12). Sin embargo, la inyección en el tejido muscular circundante produce un efecto contrario al deseado, pues se producirá una vasodilatación (13).

\section{Diseño y elevación del colgajo}

Un adecuado conocimiento del aparato gingival y submucoso es importante en la planificación del acceso a la zona quirúrgica. Es necesario tener en cuenta que los vasos sanguíneos supraperiósticos se extienden desde la mucosa alveolar, paralelos al eje longitudinal de los dientes (6). Una incisión vertical, en lugar de una incisión angulada limitará la hemorragia. La elevación del colgajo, conservando la microvasculatura, disminuirá la potencial pérdida de control de la hemorragia durante la cirugía (14).

\section{Agentes hemostáticos utilizados en cirugía periapi- cal}

Sulfato férrico

El sulfato férrico es un producto químico que se ha utilizado como agente hemostático desde que se introdujo por primera vez como solución de Monsel (subsulfato férrico al 20\%) en 1857. Es un agente necrosante con un $\mathrm{pH}$ extremadamente bajo que va entre 0,8 a 1,6 (15). Su modo de acción es el resultado de una reacción química de la sangre con los iones de hierro y sulfato para formar una aglutinación de proteínas de la sangre. El coágulo que se forma se conecta a las aberturas capilares para crear la hemostasia resultante (6).

Se aplica directamente a la superficie del hueso y la hemostasia se logra casi inmediatamente. Sin embargo,está demostrado que es citotóxico, y si no se elimina por completo de la superficie del hueso al final del procedimiento dará lugar a una inflamación severa y el posterior retraso en la cicatrización $(15,16)$.

El efecto necrosante, además de la dificultad en el control de su distribución y completa eliminación, se oponen fuertemente a su selección en áreas de interés neurovascular, como son el nervio dentario inferior, el foramen mental, el seno maxilar y el piso nasal (15-17).

La solución de sulfato férrico parece ser un agente hemostático seguro, siempre y cuando se utilice en cantidades limitadas, y se tenga el cuidado de eliminarlo completamente de la cripta ósea antes de la sutura (15). Dependiendo de la concentración del producto, está disponible comercialmente como Astringedent ${ }^{\mathbb{B}}$, ViscoStat ${ }^{\mathbb{}}$, Stasis $^{\circledR}$, Quick-Stat $^{\mathrm{TM}}$ FS y Cut-trol ${ }^{\circledR}$ (4) (Figura 1).

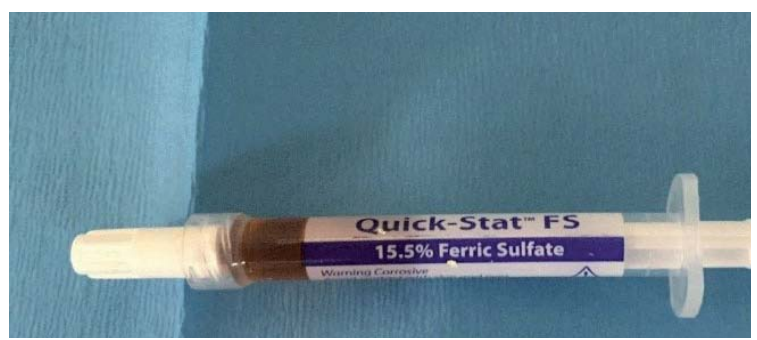

Figura 1. Quick-Stat ${ }^{\mathrm{TM}} \mathrm{FS}$, sulfato férrico en gel al $15.5 \%$

\section{Trombina}

La trombina de uso tópico, Thrombogen ${ }^{\circledR}$ fue desarrollada para proporcionar hemostasia cuando el sangrado proviene de pequeños capilares y vénulas. La trombina inicia las vías extrínseca e intrínseca de la coagulación, y actúa rápidamente al coagular directamente el fibrinógeno en la sangre (15). Está diseñada sólo para la aplicación tópica y puede ser potencialmente mortal si se inyecta. La trombina de uso tópico ha sido investigada como un agente hemostático para la disminución del sangrado en el hueso esponjoso (6).

A pesar de que la trombina de uso tópico ha sido 
utilizada con éxito en neurocirugía, cirugía cardiovascular y quemaduras, no es muy utilizada en cirugía periapical. Las principales desventajas son la difícil manipulación y el alto costo $(6,15)$.

\section{Sulfato de Calcio}

El Sulfato de Calcio (yeso París) se ha utilizado principalmente en odontología para rellenar grandes defectos óseos quirúrgicos, como material de barrera en procedimientos de regeneración tisular guiada, y también como agente hemostático $(9,17,18)$.

Se trata de un material reabsorbible que consta de un polvo y líquido que se pueden mezclar en una consistencia similar a la masilla. Se coloca en la cripta ósea utilizando bolitas de algodón mojadas para presionarlo contra las paredes $(19,20)$. El mecanismo de acción es similar a la cera ósea, que actúa como una barrera mecánica para obstruir los capilares $(9,18)$. El sulfato de calcio es biocompatible, y se reabsorbe completamente de 2 a 4 semanas, y no causa una respuesta inflamatoria a largo plazo. También tiene la ventaja de ser relativamente barato (9).

\section{Esponjas a base de gelatina}

Gelfoam ${ }^{\circledR}$ y Spongostan ${ }^{\circledR}$ son esponjas gelatinosas que son insolubles en agua y biológicamente reabsorbibles $(6,21)$. Están hechas de piel animal purificada y se vuelven blandas en contacto con la sangre. Se cree que actúan intrínsecamente mediante la promoción de la desintegración de las plaquetas, con la posterior liberación de tromboplastina y plastina. Esto a su vez, estimula la formación de trombina y soporta las hebras de fibrina de los intersticios de la esponja $(6,9)$.

Al utilizarlas, se hinchan y forman una masa blanda y gelatinosa. Esta masa puede verse oscura, por lo que puede interferir la preparación y obturación retroapical, así como también es difícil ejercer presión sobre ella. El uso principal de esponjas a base de gelatina en cirugía periapical es en la cripta ósea, antes de la sutura (7).

\section{Gasa de celulosa oxidada}

Surgicel ${ }^{\mathrm{TM}}$ es un material esterilizado químicamente que se prepara mediante la oxidación de $\alpha$-celulosa regenerada (oxicelulosa). El elemento básico es el ácido polianhidroglucurónico, que se trenza en hebras y después se teje para formar una gasa (14).

Su modo de acción es básicamente una barrera física, que inicialmente actúa sobre la sangre para formar luego una masa semejante a un coágulo. No estimula la cascada de la coagulación mediante la adhesión ni la acción de las plaquetas $(14,21)$.

Torundas de algodón impregnadas con vasoconstrictor Los vasoconstrictores han sido ampliamente recomendados como agentes tópicos para el control de la hemorragia durante la cirugía periapical. De estos, la epinefrina ha demostrado ser el más eficaz y el más recomendado $(6,7)$.

Las torundas de algodón que contienen clorhidrato de epinefrina racémica se comercializan como Epidri ${ }^{\mathrm{TM}}$, Racellet $^{\mathrm{TM}}$ y Radri ${ }^{\mathrm{TM}}$ (Figura 2). La cantidad de clorhidrato de epinefrina racémica en cada presentación varía. Por ejemplo, cada torundade Epidri ${ }^{\mathrm{TM}}$ contiene un promedio de 1,9 mg. Cada Racellet ${ }^{\mathrm{TM}} \# 2$ contiene un promedio de $1,15 \mathrm{mg}$ y cada Racellet ${ }^{\mathrm{TM}} \# 3$ contiene un promedio de $0,55 \mathrm{mg}$. Las torundas Radri ${ }^{\mathrm{TM}}$ contienen una combinación de vasoconstrictor y astringente. Cada torunda Radri ${ }^{\mathrm{TM}}$ contiene un promedio de $0.45 \mathrm{mg}$ de clorhidrato de epinefrina y un promedio de $1,85 \mathrm{mg}$ de fenol sulfonato de zinc $(7,9,15)$.

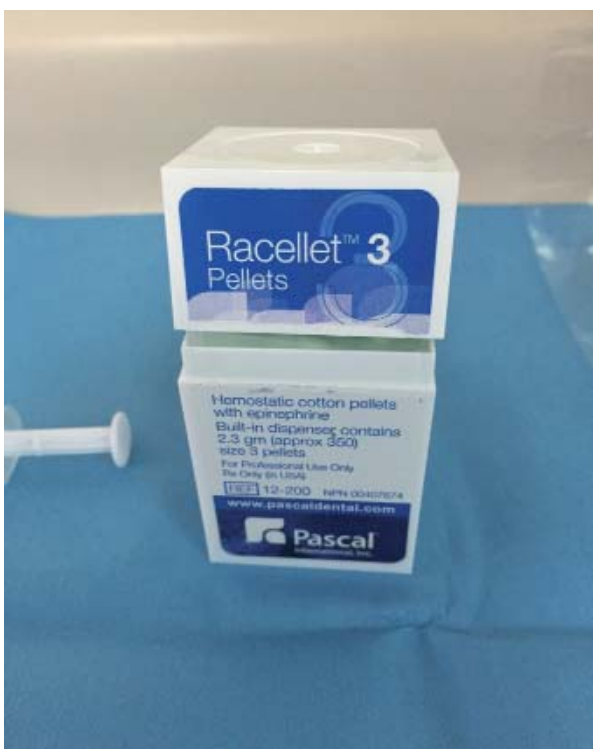

Figura 2. Torundas de algodón con epinefrina racémica Racellet $^{\mathrm{TM}} \# 3$

Una torunda impregnada se coloca sobre la cripta ósea. Las torundas no impregnadas adicionales se colocan después de la primera y se mantienen bajo presión durante 2 a 4 minutos, luego se retiran las torundas no impregnadas y se evalúa la cripta ósea para 
determinar si se ha obtenido la hemostasia adecuada.

El efecto producido es mecánico y químico, la presión proporciona la acción mecánica, y el clorhidrato de epinefrina racémica proporciona el efecto químico, causando vasoconstricción inmediata local de los vasos sanguíneos (15). Sin embargo, existen dos preocupaciones con la utilización de torundas de algodón impregnadas con epinefrina, primero, la posibilidad de que los residuos de algunas fibras de algodón queden en la cripta ósea, y segundo, el posible efecto de epinefrina racémica en el sistema cardiovascular del paciente (22-24).

\section{Cloruro de aluminio}

Hemodettes $^{\circledR}$ es un gel de cloruro de aluminio al $20 \%$ $\mathrm{y}$ es un agente soluble en agua con propiedades de aglutinación similares al sulfato férrico, pero sin los efectos secundarios perjudiciales.

Este agente se utiliza con dos torundas de algodón o un microbrush impregnado en un recipiente estéril. Su color azul hace que sea fácilmente identificable, y se puede lavar fácilmente de la cripta ósea con solución salina (4).

Expasyl ${ }^{\mathrm{TM}}$ es una pasta que contiene cloruro de aluminio y caolín, y se utiliza normalmente para producir la retracción gingival durante la toma de impresiones, sellado de prótesis, y en odontología conservadora durante restauraciones de cavidades de clase II y V (25). Recientemente, también ha sido utilizado para asegurar la hemostasia en cirugía periapical $(26,27)$.

\section{Cera para Hueso}

La fórmula de cera para hueso fabricada por Ethicon (Piscataway, NJ, USA) contiene un gran porcentaje de cera de abeja altamente purificada y un agente de ablandamiento y acondicionamiento que es el palmitato de isopropilo $(6,14)$. Ha sido utilizada principalmente por cirujanos ortopédicos y neurocirujanos para conseguir hemostasia (28).

Actúa mecánicamente cuando está firmemente en contacto con la superficie ósea y no tiene ningún efecto sobre la coagulación de la sangre. Una vez colocada en la cripta ósea, se retira un poco para exponer el ápice, y se realiza la cavidad retroapical. Completado el procedimiento toda la cera de hueso restante debe- rá ser retirada $(6,9)$. Se ha reportado que si algunos restos quedan en contacto con el hueso estos pueden ocasionar una inflamación persistente (27), reacción de células gigantes a cuerpo extraño (29), y retraso en la cicatrización (30). Por lo tanto ya no se puede recomendar su utilización porque dificulta la curación, y se dispone de varias otras alternativas (6).

\section{Agentes a base de colágeno}

La hemostasia que se consigue por medio de los agentes a base de colágeno se obtiene entre 2 a 5 minutos (9). La ventaja radica en que por lo general el control de la hemorragia es más duradero y es más predecible su efecto (4).

El mecanismo por el cual los productos a base de colágeno mejoran la hemostasia son los siguientes (6): - Estimulación de la adhesión plaquetaria, agregación plaquetaria y reacción de liberación.

- Activación del factor XIII (factor de Hageman) y posiblemente otros factores de la cascada de la coagulación.

- Taponamiento mecánico debido a la estructura que se forma en la interfaz colágeno-herida.

- Liberación de la serotonina.

Aunque Avitene ${ }^{\circledR}$ es el más conocido de esta categoría, es difícil de colocar y es costoso. Un sustituto razonable involucraría a CollaPlug ${ }^{\circledR}$ o CollaTape ${ }^{\circledR}(4)$. También están disponibles en el mercado CollaCote $\AA$, CollaStatt $₫$, Instatt $₫$ y Hemocollagene ${ }^{\circledR}(7)$. Estos agentes hemostáticos tienen un modo de acción similar, y la zona quirúrgica experimenta un patrón de curación semejante. En conjunto, los estudios de cicatrización con agentes hemostáticos a base de colágeno han mostrado resultados favorables $(4,7)$.

\section{Agentes a base de chitosan}

El chitosan es un biopolímero complejo que se deriva del exoesqueleto de los crustáceos y se ha demostrado que no es tóxico y es biodegradable. Básicamente, son aminopolisacáridos derivados semi-sintéticamente que tienen estructuras únicas, propiedades multidimensionales, funcionalidad altamente sofisticada y una amplia gama de aplicaciones en el área biomédica. Su utilidad es interesante no solo porque se produce a partir de un recurso abundante y renovable, sino porque es un biomaterial muy compatible y eficaz (31). 
La hemostasia se produce independientemente a las vías intrínseca o extrínseca de la coagulación lo cual representa una amplia ventaja, pero también las activará si están presentes (32). Los eritrocitos, leucocitos, plaquetas, bacterias y virus tienen cargas superficiales electronegativas, y se unen a este electropositivo agente hemostático, y en el caso de los eritrocitos, forman rápidamente un coágulo de sangre adhesivo (33). La presentación HemCon ${ }^{\circledR}$ ha sido investigada con buenos resultados como agente hemostático así como bacteriostático, incluso en pacientes que toman anticoagulantes $(32,34,35)$.

\section{Electrocauterización}

El cauterio interrumpe el flujo sanguíneo mediante la coagulación de la sangre y las proteínas tisulares, dejando una escara que el cuerpo intenta desprender (14). Es un método eficaz para conseguir hemostasia por coagulación. Se utiliza para detener la hemorragia localizada en los tejidos blandos, pero también se ha informado que es eficiente cuando se utiliza en superficies óseas (36).

La preocupación planteada con esta técnica es el retraso en la curación en las superficies óseas que se puede producir después de la emisión local de altas temperaturas $(7,37)$. El efecto perjudicial de la aplicación de calor al hueso es proporcional tanto a la temperatura como la duración de la aplicación (14).

\section{Láseres}

Los láseres tienen muchas indicaciones en el campo odontológico, que incluyen también el control de la hemostasia (38-40). El láser Erbio: itrio-aluminio-granate (Er: YAG) tiene una longitud de onda de $2.940 \mathrm{~nm}$ y se administra utilizando una fibra óptica sólida. Tiene una gran afinidad por el agua y la hidroxiapatita (14).

Es el preferido para el control de la hemorragia porque crea un campo seco, además tiene la capacidad de vaporizar los tejidos y cierra por coagulación pequeños vasos. También puede eliminar tejidos duros sin drásticos cambios térmicos en su estructura (41).

\section{DISCUSIÓN}

El éxito de la cirugía periapical puede verse afectado por distintos factores, y un adecuado control de la hemorragia del campo quirúrgico es sumamente esen- cial, sobre todo si se realizará microcirugía $(4,15,26)$ pues proveerá adicionalmente a la hemostasia un adecuado acceso, control del dolor y cicatrización (7). Un agente hemostático ideal debe ser estéril, de fácil aplicación, visible en la cripta ósea, no irritante, fácilmente removible y capaz de secar el campo operatorio (1).

La generación de hemostasia se consigue fundamentalmente mediante una profunda anestesia, sin embargo algunos clínicos optan por inyectar la solución anestésica por vía intraósea en el hueso esponjoso para acrecentar el efecto hemostático. Con respecto a esto, Baker y col. realizaron esta maniobra en cerdos utilizando lidocaína al 2\% con epinefrina 1:50000, comparándola con la clásica técnica infiltrativa; y evaluaron la cantidad de sangrado en tejidos blandos y duros. Ambas técnicas fueron efectivas, sin embargo algunos cerdos mostraron elevados niveles de frecuencia cardiaca después de la anestesia intraósea debido a la epinefrina. Por lo tanto los autores recomendaron utilizar solamente la anestesia infiltrativa debido a sus bajos riesgos sistémicos (12).

Particularmente, el efecto de la epinefrina para el control de la hemostasia es muy conocido, de hecho las torundas con epinefrina son los agentes más utilizados en cirugía periapical contemporánea, sin embargo la preocupación radicaría en el posible compromiso del sistema cardiovascular del paciente (23). La diferencia se basa en el modo de aplicación. Kim y Kratchman sustentan que si se utilizan por vía tópica se producirá una vasoconstricción local inmediatamente, por lo tanto, habrá poca absorción hacia la circulación sistémica, y se reducirá la probabilidad de un efecto adverso sobre el sistema cardiovascular (15).

Esto concuerda con el estudio de Besner, quien informó que la frecuencia del pulso no cambia cuando se utiliza un Racellet ${ }^{\top M}$ \#2 por vía tópica (24). Asimismo, también concuerda con Vy y col., quienes utilizaron epinefrina a través del uso de esponjas de colágeno reabsorbibles CollaCote ${ }^{\circledR}$ y tampoco detectaron eventos cardiovasculares sistémicos (23). Adicionalmente, una evaluación de cirugías periapicales con un año de control fue realizada por Peñarrocha-Diago y col., quienes compararon las torundas embebidas con epinefrina con un agente hemostático a base el cloruro de aluminio (Expasil ${ }^{\mathrm{TM}}$ ), y no hallaron diferencia significativa entre ambos (26). 
Un agente hemostático cuya utilización ha sido ampliamente difundida es el sulfato férrico, Kim y Rethnam indican que es seguro, siempre y cuando se utilice en cantidades limitadas (9). Su acción hemostática fue evaluada por Vickers y col., en un estudio con 39 pacientes ASA I o II, donde compararon el sulfato férrico al $20 \%$ con las torundas Racellet ${ }^{T M} \# 3$; $\mathrm{y}$ encontraron que ambos métodos son igualmente eficaces, demostrando de esta manera que el sulfato férrico es tan eficaz como las torundas con epinefrina (22). Sin embargo, es de considerable importancia que se tenga la certeza de eliminarlo completamente de la cripta ósea, pues en el estudio de Lemon y col. se informa un efecto adverso sobre la cicatrización ósea en mandíbulas de conejos cuando el sulfato férrico fue dejado in situ(17). En un estudio paralelo con el mismo modelo de conejos, Jeansonne y col., no encontraron diferencia significativa en la cicatrización cuando la cripta ósea se raspó y se irrigó con solución salina después de una aplicación de 5 minutos de sulfato férrico (16).

En un experimento también en conejos realizado por Azargoon y col., el sulfato férrico fue comparado con las propiedades hemostáticas de un reciente agente desarrollado a base de chitosan $\left(\operatorname{HemCon}^{\circledR}\right)$, y observaron que después de 21 días no hubo diferencia entre ambos agentes. De hecho, HemCon ${ }^{\circledR}$ mostró una mayor formación ósea (34). Asimismo, el efecto hemostático del sulfato férrico fue inferior al sulfato de calcio en el estudio de Scarano y col., realizado en modelos de conejo sometidos a cirugía periapical (18). Además, una gran diferencia radica en que el sulfato de calcio puede dejarse in situ (14).

De todos los agentes hemostáticos descritos, el más tradicional es la cera para hueso, sin embargo Johnson y col., sugieren que actualmente ya no puede considerarse de elección en cirugía periapical debido a que existen alternativas con menos efectos adversos (14), caso contrario ocurre con el sulfato de calcio que ofrece un buen efecto hemostático de acuerdo a Scarano y col., (18) y un buen resultado en defectos de espesor total de acuerdo a los estudios de Pécora y col., (19) y Murashima y col., (20). En un estudio en tibias de ratas, Ibarrola y col. evaluaron el efecto hemostático de la cera para hueso, comparada con una esponja a base de gelatina $\left(\right.$ Gelfoam $\left.^{\circledR}\right)$ y una gasa de celulosa oxidada (Surgicel ${ }^{\mathrm{TM}}$ ); y encontraron que la mejor respuesta histológica se obtuvo con Gelfoam ${ }^{\circledR}$, la cual después de 120 días se reabsorbió completamente. La cera para hueso mostró la peor respuesta histológica (21). También este agente hemostático fue evaluado en el estudio de Von Arx y col., quienes utilizaron la cera para hueso comparada con sulfato férrico $\left(\right.$ Stasis $\left.^{\mathbb{B}}\right)$, cloruro de aluminio (Expasyl ${ }^{\mathrm{TM}}$ ), y una combinación de Stasis ${ }^{\circledR}+$ Expasyl $^{\mathrm{TM}}$. Encontraron que el mejor efecto hemostático fue para Stasis ${ }^{\circledR}+\mathrm{Ex}-$ pasyl $^{\mathrm{TM}}$, y el peor para la cera para hueso (27).

Un agente hemostático introducido en la última década es el HemCon ${ }^{\circledR}$ que es a base de chitosan y los estudios son prometedores. Malmquist y col., en 17 pacientes sometidos a cirugías orales, incluso en pacientes que tomaban anticoagulantes obtuvieron rápida hemostasia dentro del primer minuto de aplicación (32) Sin embargo, en el estudio de Azargoon y col., fue observada una hemostasia primaria con sangrado intermitente en el primer minuto, y una hemostasia plena en aproximadamente 2 minutos (34).

La combinación de los agentes hemostáticos es una opción que fue evaluada por Jensen col., quienes analizaron el efecto hemostático y las reacciones óseas en defectos óseos preparados en cráneos de conejos. Para esto utilizaron Expasyl ${ }^{\mathrm{TM}}+$ Stasis $^{\circledR}{ }^{\circledR}$ Expasyl $^{\mathrm{TM}}$ + Stasis $^{\circledR}+$ refrescar el defecto óseo con una fresa, Spongostan $^{\circledR}$, Spongostan ${ }^{\circledR}+$ epinefrina, y electrocauterización. Demostraron que Expasyl ${ }^{\mathrm{TM}}+$ Sta$\operatorname{sis}^{\circledR}$ y la electrocauterización fueron las más efectivas en cuanto a control de hemorragia, sin embargo recomendaron refrescar con un fresa el defecto óseo antes de suturar, a fin de no comprometer la curación debido a las reacciones óseas desfavorables que presentaron (36). Otra combinación de agentes hemostáticos fue realizada por Vy y col., utilizando epinefrina a través del uso de esponjas de colágeno reabsorbibles CollaCote ${ }^{\circledR}$ con excelentes resultados (23)

La utilización de los láseres ha sido recomendada para muchos procedimientos odontológicos que incluyen el control de la hemorragia de acuerdo a Komori y col., (41). Sin embargo, Arnabat-Domiguez y col. en un ensayo clínico aleatorizado en 50 criptas óseas para implantes dentales utilizando el Erbio: itrio-aluminio-granate (Er: YAG) no encontraron diferencias en el control de la hemorragia, comparándolas con la segunda exposición quirúrgica convencional para la colocación de los implantes (40). 
Finalmente, independientemente del agente hemostático elegido, el clínico debe estar consciente de las propiedades biológicas que éste posea y debe prestar atención a las posibles complicaciones. Aunque una eficaz hemostasia es un requisito importante, una cicatrización adecuada de las estructuras periapicales no puede garantizarse si no hay una estabilidad biológica de los agentes utilizados.

\section{CONCLUSIONES}

Los odontólogos al realizar cirugía o microcirugía periapical deben estar conscientes de los posibles problemas que acarrearían de no controlar la hemorragia durante los procedimientos quirúrgicos, $\mathrm{y}$ deben ser capaces de anticiparse a esta situación a través del desarrollo de una correcta historia médico-odontológica, así como disponer de los materiales necesarios que hagan su trabajo mucho más efectivo y seguro. La utilización de agentes hemostáticos constituye una imperativa necesidad a fin de obtener un campo operatorio seco y libre de contaminación que permita realizar adecuadamente los procedimientos quirúrgicos actuales.

Dentro de los agentes hemostáticos tradicionales, la cera para hueso ya no es actualmente recomendable debido a sus efectos adversos, a diferencia del sulfato de calcio y e sulfato férrico. Así mismo, las torundas de algodón con epinefrina constituyen el agente hemostático más utilizado en cirugía periapical contemporánea.

La combinación de uno o más agentes hemostáticos también puede representar una alternativa en base a la literatura analizada.

\section{Correspondencia:}

Eric Hernán Coaguila Llerena

Teléfono: 51958952576

Correo electrónico: ehernanco@gmail.com

\section{REFERENCIAS BIBLIOGRÁFICAS}

1. Sauveur G, Roth F, Sobel M, Boucher Y. The control of haemorrhage at the operative site during periradicular surgery. Int Endod J. 1999; 32(3):225-8.

2. Rubinstein R, Torabinejad M. Contemporary endodon- tic surgery. J Calif Dent Assoc. 2004; 32(6):485-92.

3. Kim S. Modern endodontic practice: instruments and techniques. Dent Clin North Am. 2004; 48(1):1-9.

4. Niemczyk SP. Essentials of endodontic microsurgery. Dent Clin North Am. 2010; 54(2):375-99.

5. Selim HA, el Deeb ME, Messer HH. Blood loss during endodontic surgery.Endod Dent Traumatol. 1987; 3(1):33-6.

6. Witherspoon DE, Gutmann JL. Haemostasis in periradicular surgery. Int Endod J. 1996; 29(3):135-49.

7. Hargreaves KM, Khan A. Surgical preparation: anesthesia \& hemostasis. Endod Topics. 2005; 11: 32-55.

8. Jang Y, Kim E. Cardiovascular effect of epinephrine in endodontic microsurgery: a review. Restor Dent Endod. 2013; 38(4):187-93.

9. Kim S, Rethnam S. Hemostasis in endodontic microsurgery. Dent Clin North Am. 1997; 41(3):499-511.

10. Wali M, Drum M, Reader A, Nusstein J. Prospective, randomized single-blind study of the anesthetic efficacy of 1.8 and 3.6 milliliters of $2 \%$ lidocaine with 1:50,000 epinephrine for inferior alveolar nerve block. J Endod. 2010; 36(9):1459-62.

11. Gutmann JL, Frazier LW Jr, Baron B. Plasma catecholamine and haemodynamic responses to surgical endodontic anaesthetic protocols. Int Endod J. 1996; 29(1):37-42.

12. Baker TF,Torabinejad M,Schwartz SF, Wolf D. Effect of intraosseous anesthesia on control of hemostasis in pigs. J Endod. 2009; 35(11):1543-5.

13. Milam SB, Giovannitti JA Jr. Local anesthetics in dental practice. Dent Clin North Am. 1984; 28(3):493508.

14. Johnson BR, Fayad MI, Witherspoon DE. Cirugía periradicular. En: Hargreaves KM, Cohen S. Vías de la Pulpa. 10ma ed. Barcelona: Elsevier; 2011.p.720-68.

15. Kim S, Kratchman S. Modern endodontic surgery concepts and practice: a review. J Endod. 2006; 32(7):601-23.

16. Jeansonne BG, Boggs WS, Lemon RR. Ferric sulfate hemostasis: effect on osseous wound healing. II. With curettage and irrigation. J Endod. 1993; 19(4):174-6.

17. Lemon RR, Steele PJ, Jeansonne BG. Ferric sulfate hemostasis: effect on osseous wound healing. Left in situ for maximum exposure. J Endod. 1993; 19(4):170-3.

18. Scarano A, Artese L, Piattelli A, Carinci F, Mancino C, Iezzi G. Hemostasis control in endodontic surgery: a comparative study of calcium sulfate versus gauzes and versus ferric sulfate. J Endod. 2012; 38(1):20-3.

19. Murashima Y, Yoshikawa G, Wadachi R, Sawada N, Suda H. Calcium sulphate as a bone substitute for various osseous defects in conjunction with apicectomy. Int Endod J. 2002;35(9):768-74.

20. Pecora G, De Leonardis D, Ibrahim N, Bovi M, Cornelini R. The use of calcium sulphate in the surgical treatment of a 'through and through' periradicular le- 
sion. Int Endod J. 2001;34(3):189-97.

21. Ibarrola JL, Bjorenson JE, Austin BP, Gerstein H. Osseous reactions to three hemostatic agents. J Endod. 1985; 11(2):75-83.

22. Vickers FJ, Baumgartner JC, Marshall G. Hemostatic efficacy and cardiovascular effects of agents used during endodontic surgery. J Endod. 2002; 28(4):3223.

23. Vy CH, Baumgartner JC, Marshall JG. Cardiovascular effects and efficacy of a hemostatic agent in periradicular surgery. J Endod. 2004; 30(6):379-83.

24. Besner E. Systemic effects of racemic epinephrine when applied to the bone cavity during periapical surgery. Va Dent J. 1972; 49(5):9-12.

25. Shannon A. Expanded clinical uses of a novel tissue-retraction material. Compend Contin Educ Dent. 2002; 23(S1):3-6.

26. Peñarrocha-Diago $M$, Maestre-Ferrín L, Peñarrocha -Oltra D, von Arx T, Peñarrocha-Diago M. Influence of hemostatic agents upon the outcome of periapical surgery: dressings with anesthetic and vasoconstrictor or aluminum chloride. Med Oral Patol Oral Cir Bucal. 2013;18(2):e272-8.

27. von Arx T, Jensen SS, Hänni S, Schenk RK. Haemostatic agents used in periradicular surgery: an experimental study of their efficacy and tissue reactions. Int Endod J. 2006; 39(10): 800-8.

28. Finn MD, Schow SR, Schneiderman ED. Osseous regeneration in the presence of four common hemostatic agents.J Oral Maxillofac Surg. 1992; 50(6):608-12.

29. Wolvius EB,van der Wal KG. Bone waxas a cause of a foreign body granuloma in a cranial defect: a case report. Int J Oral Maxillofac Surg. 2003; 32(6):656-8.

30. Xu Y,Sun J,Chen Z. Establishment of a rat model for alveolar cleft with bone wax. J Oral Maxillofac Surg. 2015; 73(4):733.

31. Dash M, ChielliniF, Ottenbrite RM, ChielliniE. Chitosan A versatile semi-synthetic polymer in biomedical applications. Progress in Polymer Science. 2011; 36(8):981-1014.

32. Malmquist JP, Clemens SC, Oien HJ, Wilson SL. Hemostasis of oral surgery wounds with the HemCon Dental Dressing. J Oral Maxillofac Surg. 2008; 66(6):1177-83.

33. Yang J, Tian F, Wang Z, Wang Q, Zeng YJ, Chen SQ. Effect of chitosan molecular weight and deacetylation degree on hemostasis. J Biomed Mater Res B Appl Biomater.2008;84(1):131-7.

34. Azargoon H, Williams BJ, Solomon ES, Kessler HP, He J, Spears R. Assessment of hemostatic efficacy and osseous wound healing using Hem Con dental dressing.J Endod. 2011;37(6):807-11.

35. Pippi R, Santoro M, Cafolla A. The effectiveness of a new method using an extra-alveolar hemostatic agent after dental extractions in older patients on oral anti- coagulation treatment: an intrapatient study. Oral Surg Oral Med Oral Pathol Oral Radiol. 2015;120(1):1521.

36. Jensen SS, Yazdi PM, Hjørting-Hansen E, Bosshardt DD, von Arx T. Haemostatic effect and tissue reactions of methods and agents used for haemorrhage control in apical surgery.Int Endod J. 2010; 43(1):57-63.

37. Eriksson A, Albrektsson T, Grane B, McQueen D. Thermal injury to bone: A vital-microscopic description of heat effects.Int J Oral Surg. 1982; 11(2):11521.

38. Miserendino LJ. The laser apicoectomy: endodontic application of the $\mathrm{CO} 2$ laser for periapical surgery. Oral Surg Oral Med Oral Pathol. 1988; 66(5):615-9.

39. Barak S, Katz J, Kaplan I. The use of the CO2 laser in oral surgery in the military. J Clin Laser Med Surg. 1992; 10(1):31-5.

40. Arnabat-Domínguez J, España-Tost AJ, Berini-Aytés L, Gay-Escoda C. Erbium: YAG laser application in the second phase of implant surgery: a pilot study in 20 patients. Int J Oral Maxillofac Implants.2003; 18(1):104-12.

41. Komori T, Yokoyama K, Takato T, Matsumoto K. Clinical application of the erbium:YAG laser for apicoectomy. J Endod. 1997; 23(12):748-50.

Recibido : 10/11/2014

Aceptado: 07/02/2015 